\title{
Fractal Topological Analysis for 2D Binary Digital Images
}

\author{
S. Blanco-Trejo • C. Alemán-Morillo · F. Díaz-del-Río · P. Real
}

Received: 2 January 2018 / Revised: 15 May 2018 / Accepted: 25 May 2018 / Published online: 15 October 2018

(C) Springer Nature Switzerland AG 2018

\begin{abstract}
Fractal dimension is a powerful tool employed as a measurement of geometric aspects. In this work we propose a method of topological fractal analysis for 2D binary digital images by using a graph-based topological model of them, called Homological Spanning Forest (HSF, for short). Defined at interpixel level, this set of two trees allows to topologically describe the (black and white) connected component distribution within the image with regards to the relationship "to be surrounded by". This distribution is condensed into a rooted tree, such that its nodes are connected components determined by some special sub-trees of the previous HSF and the levels of the tree specify the degree of nesting of each connected component. We ask for topological auto-similarity by comparing this topological description of the whole image with a regular rooted tree pattern. Such an analysis can be used to directly quantify some characteristics of biomedical images (e.g. cells samples or clinical images) that are not so noticeable when using geometrical approaches.
\end{abstract}

Keywords Digital image $\cdot$ Region-adjacency tree $\cdot$ Fractal topology $\cdot$ Homological spanning forest

Mathematics Subject Classification 54A05 $\cdot 54 \mathrm{~A} 10 \cdot 54 \mathrm{D} 80 \cdot 54 \mathrm{~F} 65 \cdot 54 \mathrm{H} 20$

\section{Introduction}

In biomedical images, it is common to find structured data that does not seem to respond to geometric organizational patterns, but to relational patterns between different subgroups of data [11,14,21].

This work has been supported by the Spaniard research projects (supported by the Ministerio de Economía y Competitividad and FEDER funds) COFNET (Event-based Cognitive Visual and Auditory Sensory Fusion, TEC2016-77785-P) and TOP4COG (Topological Recognition of 4D Digital Images via HSF model, MTM2016-81030-P (AEI/FEDER,UE)).

S. Blanco-Trejo · C. Alemán-Morillo · F. Díaz-del-Río · P. Real ( $₫)$

HTS. Informatics Engineering, Avda. Reina Mercedes, s/n, Seville, Spain

e-mail: real@us.es

F. Díaz-del-Río

e-mail: fdiaz@us.es

S. Blanco-Trejo

e-mail: sblanco1@us.es 


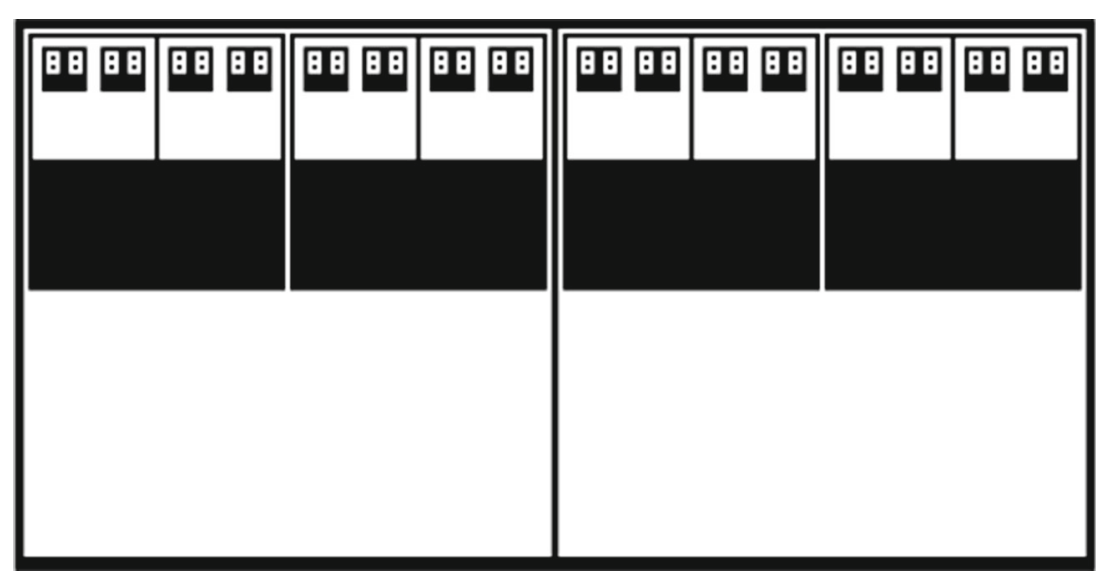

Fig. 1 A synthetic fractal image created by using a MATLAB script

This type of behavior is abundant when dealing with organic structures. E.g: the same organelle type from two different cells shall present similar geometric properties, but not equal. However, it must keep the same connectivity relationships with the remaining organelles of the same cell in order to perform its functions. Other examples would be the organs of the body, which may differ slightly in their shape from one person to another, but they must keep their connectivity relationships with other organs in order to maintain their correct function. This fact allows the organ recognition without taking into account geometrical features. For this reason, in order to be able to analyze these non-geometric pattern recognition, we develop here a pure topological approach. Another important fact that can be found in many natural phenomena, is that their structures frequently exhibit repetition of the same patterns at different scales. This type of multi-scale repetition effects is extensively studied at geometric level in fractal geometry $[4,10]$. A synthetic fractal is depicted at Fig. 1.

In this article we propose a method to perform a fractal topological analysis applied to 2D biomedical images, analyzing phenomena of non-geometric fractality in 2D binary images. In order to do that we use the HSF (Homological Spanning Tree) method that provides us topological information about the black and white connected components of the image and the relationships between them. The main advantage of this method lies on its computation speed, since it is thought to be parallelizable. A graph/tree representation is generated, so we can analyze the degree of repetition of the structures and the topological connections. Finally, we try to reflect this information in a parameter that we call "index of topological fractality", which can be useful in the characterization of images or description of patterns or textures.

\section{Methods for Fractal Dimension Computation}

There are several ways to compute the fractal dimension $D$ of an image. Classically it has been used the Hausdorff dimension of an image [8], but other approaches has been developed, such as Prism Counting, Epsilon-Blanket and others, as is shown in [1].

Nevertheless, as far as we can find in the literature, when it comes to computing the fractal dimension of biomedical images, most of the developed algorithms are based on a Box-Counting approach [3,9, 18-20].

The most popular Box-Counting Method (BCM), is the developed by Voss [22]. It consists on the use of a finite number of nonempty boxes $N(\delta)$ (which have a given length $\delta$ ) required to cover the surface of a structure. This method is implemented normally by covering an object with grids of different mesh size. The size of the structure can be computed by taking into account those boxes that intercept the structure for a selected mesh-size $\delta$. Computing different estimations changing the grid-size, a set of results are obtained. This allows to get a relation between the size of the structure $\left(N_{\delta}\right)$ and the size of the grid used $(\delta)$, which can be considered as an estimation of the fractal 
Fig. 2 a A fragment from north of Great Britain. b A rectangle

Fig. 3 a Box-counting fractal index computation from Fig. 2a. b

Box-counting fractal index computation from Fig. $2 b$.

Although they are topologically the same object, due to the dependence of geometry of the Box-counting method, the resulting values are different for both pictures. These values were obtained by using the ImageJ software tool for Box-Counting dimension computation a

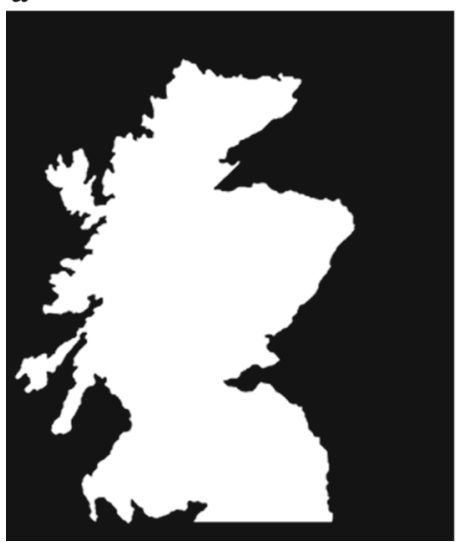

b

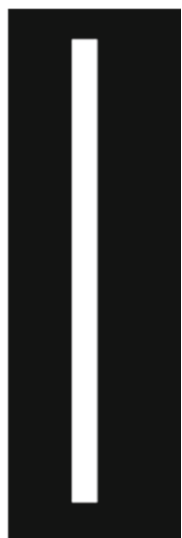

$\mathbf{a}$

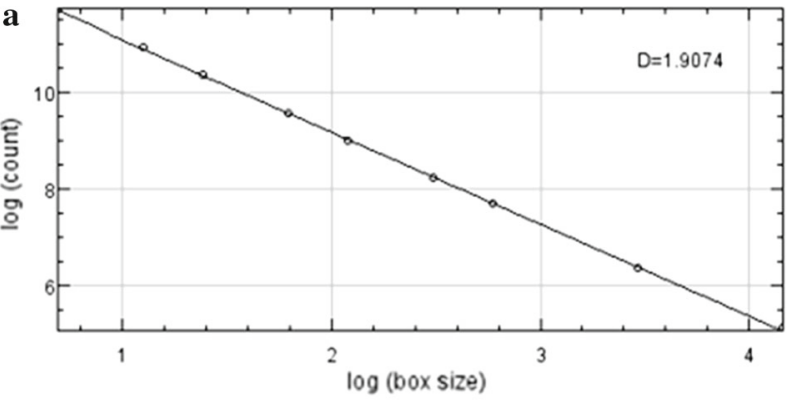

b

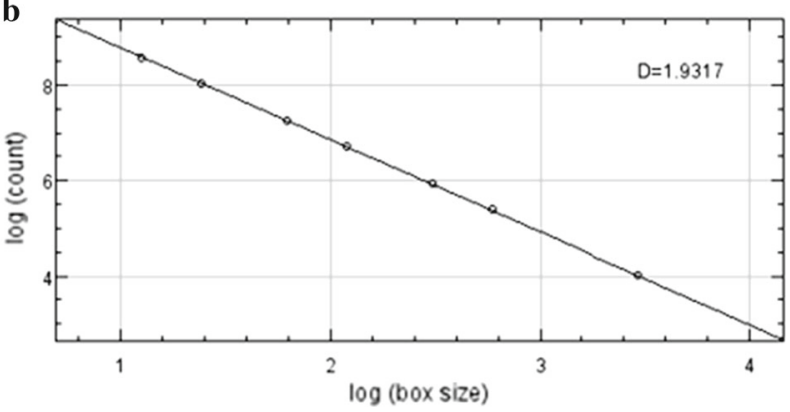

dimension. The slope $\beta$ of the logarithmic plot of the number of boxes used to cover the fractal against the box size yields to the fractal dimension $D=-\beta$ The equation for the fractal dimension computation is:

$D=-\lim _{\delta \rightarrow 0} \frac{\ln N_{\delta}(F)}{\ln (\delta)}$,

where $F$ is a non empty bounded subset of $R^{n}$ [2].

Figures 2 and 3 can be an useful example of how two images, which have the same topology, exhibit different results when computing the fractality of both of them using the Box-Counting method. The main advantage of the solutions based on this method lies on its simple, but robust way to obtain features about the geometry of the cells, and then to be able to detect anomalies regarding healthy, normal tissues [5].

On the other hand, whereas these kinds of approaches take into account the shape of the visual texture, the proposed method in this paper is based exclusively on the use of its topological properties. In other words, it performs an analysis of the global connectivity of the set of regions of constant color via the study of local connectivity between cells. This can be reached by means of the Homological Spanning Forest (HSF) computation of the digital image. 


\section{Homological Spanning Forest and Nesting Degree of the Regions}

A Homological Spanning Forest (HSF) of a binary digital image is a flexible topological model, which is composed by a set of trees "covering" it at interpixel level. In our representation, we describe an image $I$ as a cell complex whose 0-cells are the pixels of the image. In order to compute the HSF over the whole interpixel scenario of a binary image, the following steps must be followed:

1. Symmetric pACC (primal-dual Abstract Cell Complex) computation takes place, which contains information about the adjacencies between contiguous pixels.

2. MrSF (Morse Spanning Forest) computation is carried out, thus obtaining an asymmetric pACC, which is a kind of topological segmentation of the contractible cell complex.

3. Crack transports take place at the MrSF in order to create a new MrSF in which the number of primal contacts between cells of the foreground are maximized.

Physical pixel are considered here as 0-cells of the pACC, and 4-connectivity is employed for determining topological properties of the image. Sequential and parallel algorithms for computing HSFs of digital objects were developed in the works $[12,13,15]$.

An HSF $\left\{T_{1}, T_{2}\right\}$ of $I$ is represented by two trees: one $T_{1}$, "spanning" the 0 -cells of $I$ and the other $T_{2}$ "spanning" the 2-cells of $I$. Due to the fact that the image is contractible (i.e. it is topologically reducible to one point), the number of 0 -cell vertices in $T_{1}$, is one more than its number of 1 -cell vertices, and the number of 1 -cell vertices in $T_{2}$ is equal to its number of 2-cell vertices:

$$
\begin{aligned}
n^{\prime} & =n_{0}-1 \\
n_{2} & =n^{\prime \prime}{ }_{1} \\
n_{1} & =n^{\prime}{ }_{1}+n^{\prime \prime}{ }_{1}
\end{aligned}
$$

where $n_{0}$ is the number of 0 -cells, $n_{1}$ is the number of 1-cells and $n_{2}$ is the number of 2-cells in the cellularized image. In particular, we have that $n_{0}-n_{1}+n_{2}=1$

In $T_{1}$, the number of "adjacent" (through 1-cells) 0 -cells with the same color is maximized. This allows to perform segmentations of binary images, providing information about the different connected components and existing holes in the image.

Figure 4a shows an example of the HSF of a simple image. 0-cells are depicted by dots, 1-cells by triangles and 2-cells by squares. The maximal (so-called critical) sub-trees of $T_{1}$ joining adjacent 0 -cells of the same color (black or white) are connected components (CC) represented by the 0-cells 21, 26 and 31. The 1-cells labeled by 6 and 11 specify the one-dimensional holes of the image. In fact, these holes are delineated using the maximal (critical) sub-trees of $T_{2}$ joining adjacent (through 2-cells) 1-cells of the same color (in this case, black-white or white-black).

When dealing with binary images, it is easy to show that each critical 0-1 sub-tree of a CC is referred to the environment that surrounds this CC. On the other hand, each critical 1-2 sub-tree gives information about the holes in the image. Hence, the critical sub-trees allow to know the topological distribution of the image in terms of a graph structure called Region Incidence Tree (or, RIT for short). The RIT have the topological information from both 0-1 and 1-2 subtrees's critical cells, and this allows us to know how regions interact one with each other and to graphically represent it by a tree. By appropriately performing a pruning process (deleting all redundant information from the RIT of the kind "(black or white) 4-hole = (white or black) 8-CC)", we obtain the Region Adjacency Tree (or, RAT for short) (see Fig. 4b). The result of this pruning consists on preserving only the information provided by the critical 0-1 sub-trees. The level in the rooted RAT of a 4-CC provides information about its degree of nesting with regards the relationship "to be surrounded by". Let us note that the RAT is a classical notion in the image analysis literature, also called topological, homotopy or inclusion tree $[16,17]$. The root of this tree is the dummy region, an artificial region that embeds the whole image. A flowchart for obtaining the RAT is shown in Fig. 5. 
Fig. 4 a Output of an HSF computation for an image composed of three concentric regions. The $0-1$ cells tree (T1) links are represented by red (primal) and blue (dual) colors. The $1-2$ cells tree (T2) links are represented by blue (primal) and green (dual) colors. b Graphical representation of the RAT (color figure online)

\section{$\mathbf{a}$}

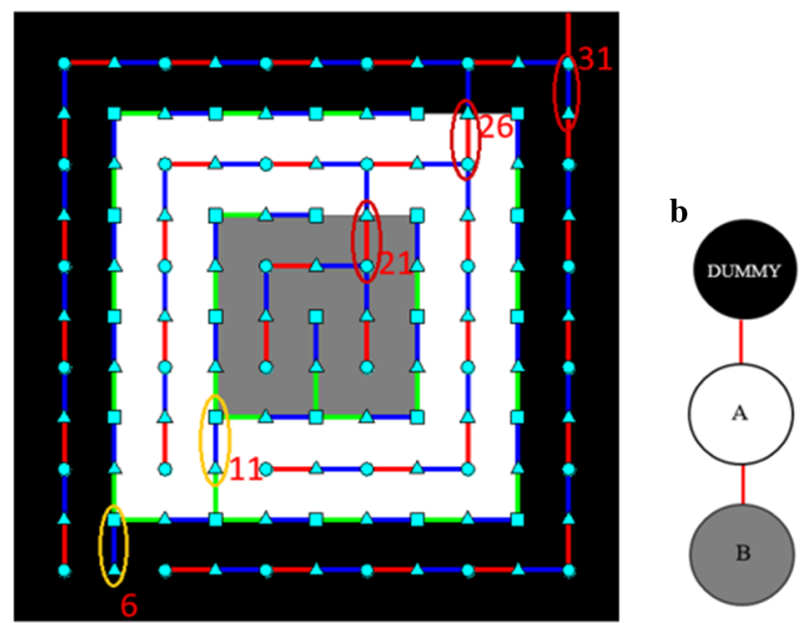

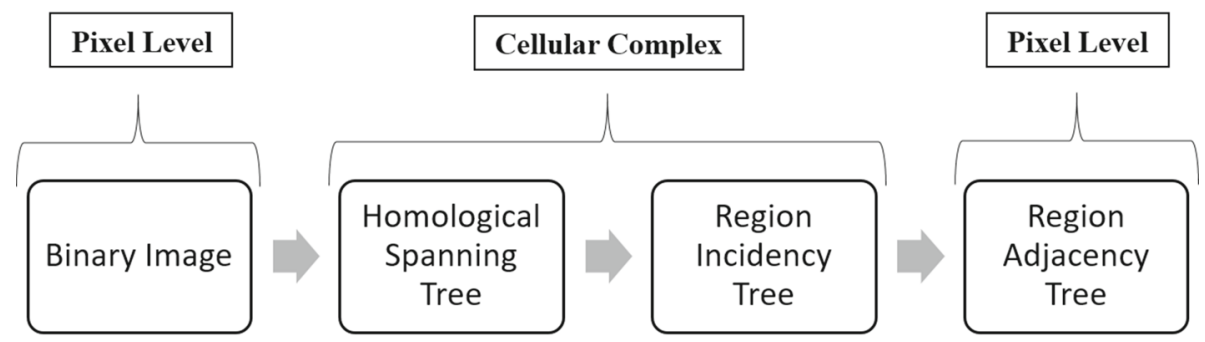

Fig. 5 A flowchart for obtaining the RAT. First, HSF is computed from a cell complex version of the binary image. Once this is achieved, the RIT can be built up from the information provided by the critical sub-trees of the HSF. Finally, the RAT is computed performing a pruning at pixel level over the RIT

In summary, the principal advantage that RAT offers for a two-dimensional binary image is that it specifies the degree of nesting for any region (4-CC) of the image with regards the topological relationship "to be surrounded by".

For gray-scale images it would be more suitable to use the RIT approach instead of the RAT. This is due to the fact that the first one contains not only information about adjacencies between regions, but also about incidences between them.

\section{Fractal Topological Analysis from HSF Representation}

In this section, we propose a first definition of topological auto-similarity and a preliminary approach for its analysis. This approximation is based on the fact that for a repetitive occurrence of a fractal structure, there must be at least some regularity in the relationships between the various related connected components of the image. Therefore, the regularity expressed in this new approach serves as a sufficient condition for the existence of fractality. Let us consider an image presenting a high degree of auto-similarity at topological level. In the case of planar images this would suppose that the topological magnitudes of a coarse or big-scaled object repeat when a zoom is applied to it. For example, if a region contains two holes, each of them must contain also two holes, and this can be extended to further zooms inside each of these inner holes. This happens for a nesting level of two in Fig. 6a. The RAT computation (such as the example depicted at the Fig. 6 b) serves not only to have an idea of how topologically nested an image is, but also to build a method of fractal dimension analysis from a pure topological point of view. 
$\mathbf{a}$

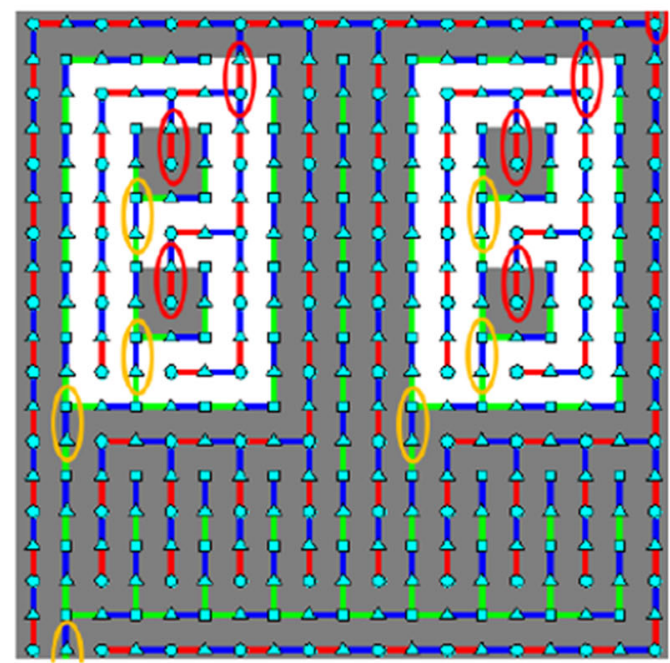

b

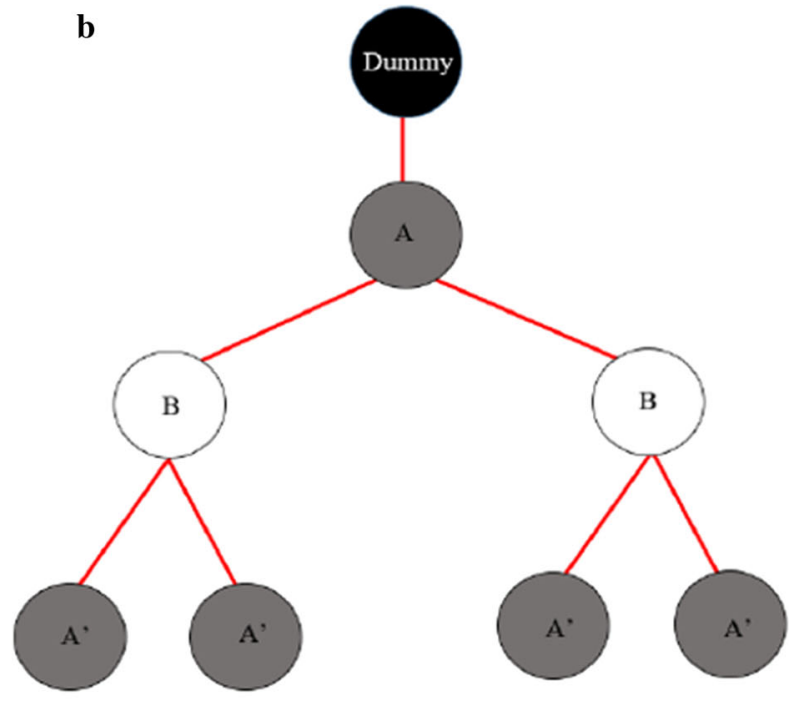

Fig. 6 a Output from a HSF computation for a fractal image. 0-cells from the critical 0-1 sub-tree are marked with red ovals, whereas critical 1-cells from the 1-2 sub-tree are marked with yellow ovals. b A representation of the inherent fractality of the image is shown in a. As can be seen, the deeper the image is being analyzed, a greater degree of detail is obtained from the tree. In this case, only critical $0-1$ sub-trees are necessary to obtain the complete topological information of the image (color figure online)
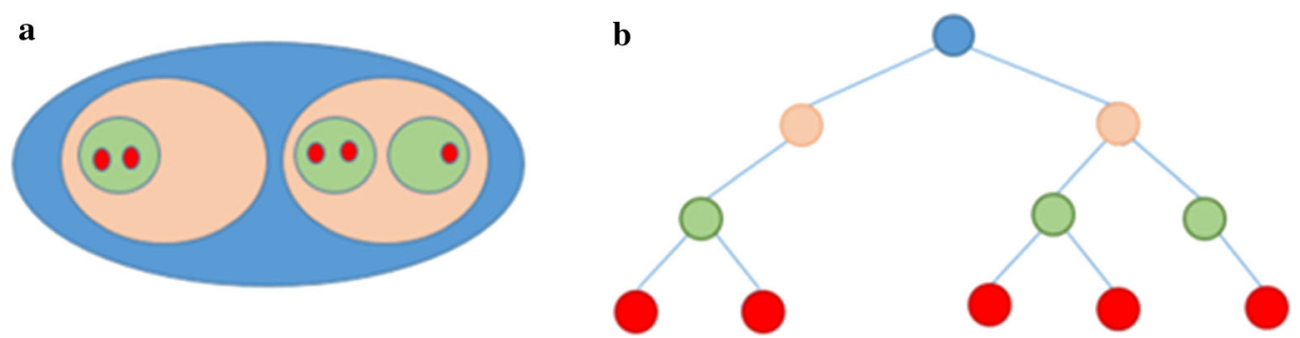

Fig. 7 a An example. Each level of depth has its own color. b The representation of the continuous image as a topological tree, where each node is a connected component (color figure online)

Once the RAT is obtained, fractal topological analysis can be carried out. The first step into our algorithm is to define a convenient "sub-tree-size" $r$ to be analyzed. Unlike geometrical methods, this size definition means how deep we are analyzing the object. This order of depth or nesting is provided naturally by the levels of the rooted RAT. To understand the order of depth we explain the example shown in Fig. 7.

A simple way to study topological auto-similarity of an image is by establishing a regular tree pattern of reference for comparing it with the RAT of the image. The rooted tree of reference that we use here is the one that has a constant number of child nodes for all its nodes. In the previous example at Fig. 7, we can see that the maximum number of components within the same connected component is two (i.e. the maximum child nodes for any node of the whole tree), so this is the value to consider. The standard tree of reference (considered here without fractal "imperfections") is that of Fig. 8b.

By comparing the reference tree with the one to be studied, we can extract their topological fractal differences (Fig. 9a). These differences are missing components as well as critical $0-1$ sub-trees derived from the missing critical 0-1 sub-tree of higher levels (Fig. 9b). The final values for each level are summarized in Fig. 10. 

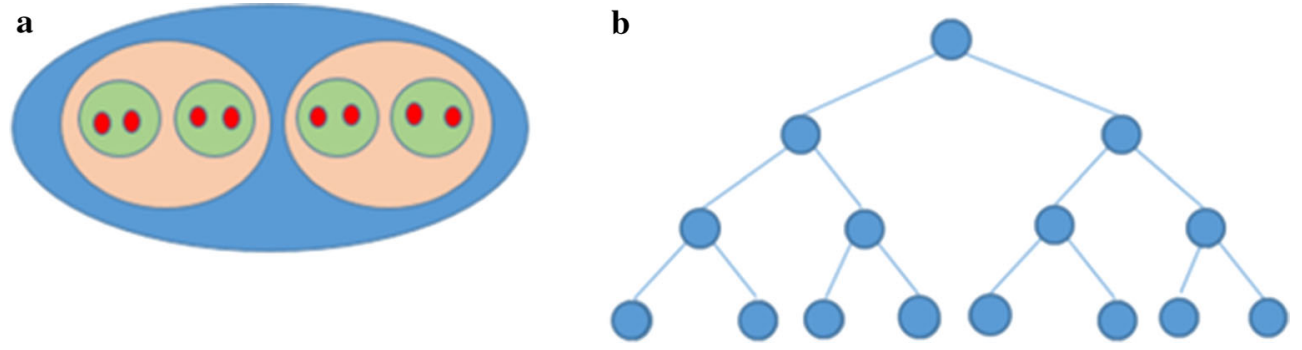

Fig. 8 a A regular system of reference. b The same system as a tree of depth

a

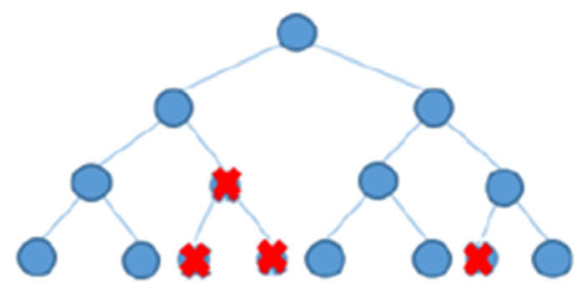

b

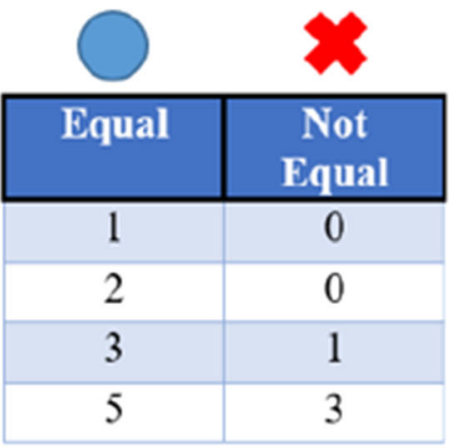

Fig. 9 a Differences between our regular system used as a pattern and the example system. b Different number of connected components between the example and the standard system

\begin{tabular}{|c|c|c|c|}
\hline Level & Equal & Total Regular Tree & Rn \\
\hline 0 & 1 & 1 & $1^{\text {* }}$ \\
\hline 1 & 2 & 2 & 1 \\
\hline 2 & 3 & 4 & 0.792 \\
\hline 3 & 5 & 8 & 0.774 \\
\hline
\end{tabular}

Fig. 10 Summary table needed to determine the fractal index for each level of depth. The depth level is showed at first column. The number of nodes for a given depth level and the total number of nodes for a given level are the parameters of the second and third columns, respectively. At fourth column, fractal indexes are shown. (*)For the zero level, the trivial case (without any perturbation) is obtained

For each level $r$, we compute the topological fractal index $F_{r}$ as the quotient between the logarithm of the number of nodes $N_{r}$ in the studied tree and the logarithm of the total number of nodes $N_{t} r$ for the reference tree.

$F_{r}=\frac{\log N_{r}}{\log N_{t r}}$

Representing the fractal index for each level $F_{r}$ with respect to its level $r$, a regression line is obtained. The slope of this line indicates the topological regularity of the image. A constant slope of value 0 indicates a totally regular figure. Conversely, the more negative the value of the slope is, the more topologically irregular the image is. Figure 11 shows the result and the regression line for the previous example.

The image from Fig. 12 used as an example seems to be a perfect topological regular image. Nevertheless, topological properties are not easy to perceive by a quickly inspection. The comparison of the RAT of the image with that of the reference tree (see Fig. 6) can be used as a necessary condition for determining topological fractality. The process described in previous section reveals the next results. Firstly, a total of $71 \mathrm{CCs}$ are found when building the HSF. Only 11 CCs shows a perfect topological regularity up to deep level 2 in the sense that the subtree rooted at those nodes and including only its children and grandchildren is the binary tree at that deep level. These CCs 
Fig. 11 Graphic representation of the fractality indexes for each level versus the associated depth level. Their regression line is also shown. The slope of this line indicates the topological regularity of the image
1,2

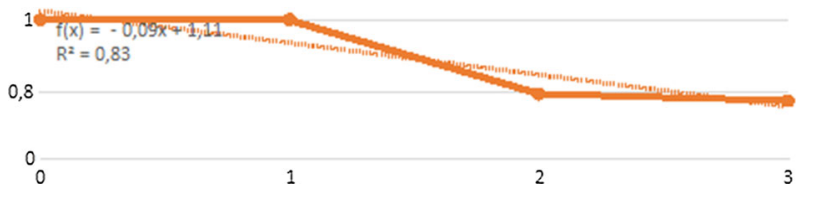

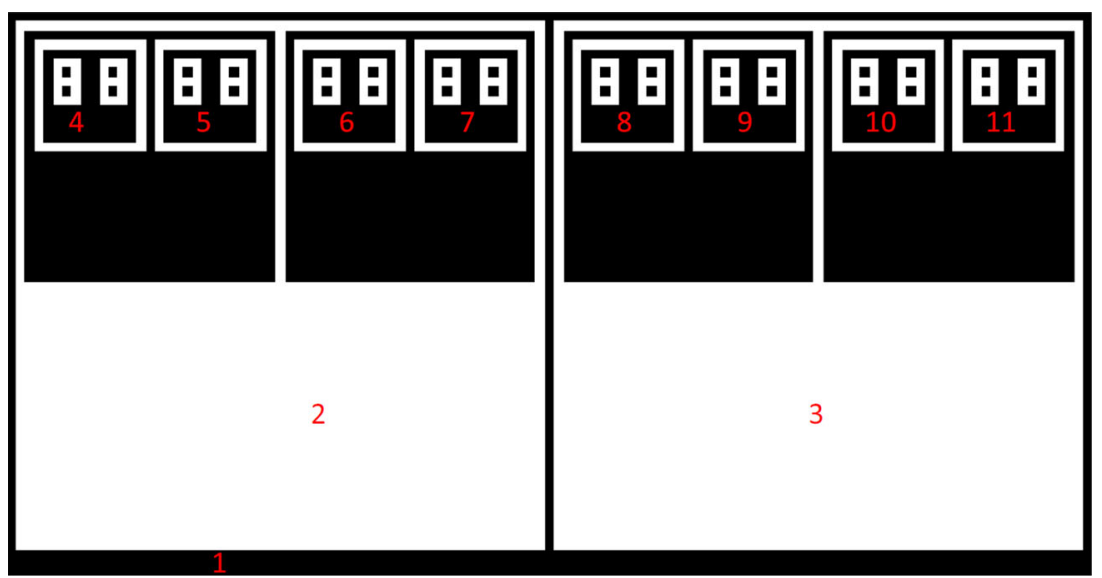

Fig. 12 A false synthetic fractal created by modifying the MATLAB script used for Fig. 1

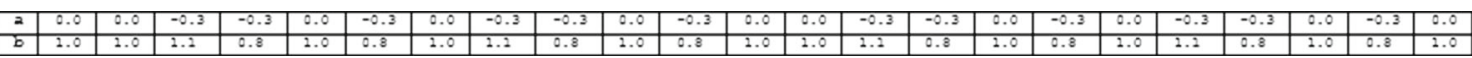

Fig. 13 Slope $a$ and coefficient $b$ of the regression lines $a x+b$ for the 23 holes that have a deep level of two at least in Fig. 12

are marked with the red numbers in Fig. 12. This pattern recognition is missed using the slope and coefficient for regression lines (Fig. 13): only those holes whose slope and coefficient are exactly 1.0 are perfect fractals. Indeed, the problem occurs with the white CCs that surround regions 4-11, because they have only one black region inside. In fact, a detailed visual inspection reveals that Fig. 12 is different from Fig. 1.

\section{Application in the Biomedical Field}

From a topological point of view, image analysis allows to easily identify those topologically equivalent structures that, conversely, can be very dissimilar at a geometric level. Practical applications can be found in many organic structures. The study of CC inclusion relationships leads to straightforward pattern analysis for those equivalent but changing structures such as cells and their content. A cell can be thought as a CC that contains other CCs (which are cell organelles). Real tissue organelles are very variable in size and shape and can be randomly distributed inside the cell. An example of topological analysis application is that of cellular blood inspection. Each type of blood cell has specific topological characteristics such as the number of nuclei, although the shape of these nuclei can vary greatly from one to another cell of the same type. This is especially relevant in the case of leukocytes or red blood cells. In Fig. 14 the cross section of spermatozoa from the caddis fly (Polycentropus) is illustrated, showing the microtube organization of motile flagella. After applying the convenient thresholding, their biological structures are reduced to a RAT, which allows us to differentiate these structures from another cells.

Other useful applications are found in the field of topological pattern recognition which can be extended for a wide selection of fields, such as biomaterials surface analysis or malignant tumor detection [6,7]. 


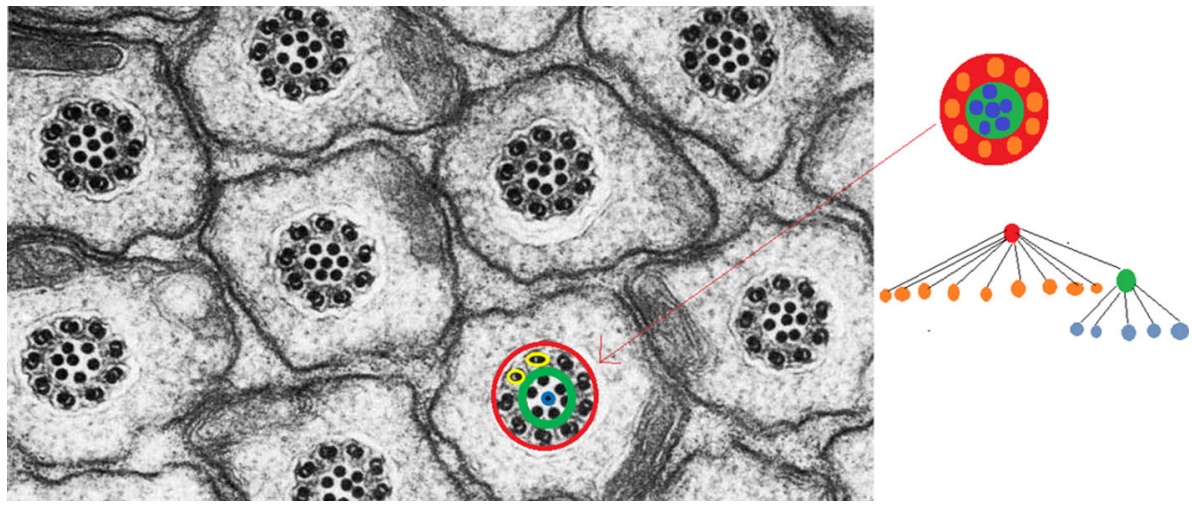

Fig. 14 Cross section of spermatozoa from the caddis fly (Polycentropus). Every tree level shows the connected components that belong to a given region. Image: Don W. Fawcett, David Phillips. The Cell Image Library, CIL:35970. Available at http://www.cellimagelibrary. org

\section{Conclusion}

This paper proposes a preliminary approach of studying topological auto-similarity for binary 2-dimensional images. Based on this, fractal topological index can be defined so that we can measure and distinguish straightforwardly the topological auto-similarity of different structures (compared with that of a reference pattern) that cannot be easily differentiated by geometric measures. The proposed fractal topological approach is only a first step towards other more elaborated descriptions of topological nature, and must be improved in the near future. An interesting line of practical development is the integration in this framework of statistical measures that allow us to describe in a more detailed way the depth of the topological trees (like the RAT). We do not look only for the number of elements per level, but also for their topological distribution. In addition, it is expected in the near future to perform this topological analysis using real gray-scale or color images, in order to compare the efficiency of our approach with other related works used for texture quantification and segmentation of biomedical images.

\section{References}

1. Annadhason, A.: Methods of fractal dimension computation. IRACST Int. J. Comput. Sci. Inf. Technol. Secur. (IJCSITS) 2, 166-169 (2012)

2. Ai, T., Zhang, R., Zhou, H.W., Pei, J.L.: Box-counting methods to directly estimate the fractal dimension of a rock surface. Appl. Surf. Sci. 314, 610-621 (2014)

3. Baish, J.W., Jain, R.K.: Fractals and cancer. Cancer Res. 60(14), 3683-3688 (2000)

4. Boeing, G.: Visual analysis of nonlinear dynamical systems: chaos, fractals, self-similarity and the limits of prediction. Systems 4(4), 37 (2016)

5. Chan, A., Tuszynski, J.A.: Automatic prediction of tumor malignancy in breast cancer with fractal dimension. R. Soc. Open Sci. 3(12), 160558 (2016)

6. Diaye, M., Degeratu, C., Bouler, J.M., Chappard, D.: Biomaterial porosity determined by fractal dimensions, succolarity and lacunarity on microcomputed tomographic images. Mater. Sci. Eng. C 33(4), 2025-2030 (2013)

7. Dobrescu, R., Vasilescu, C., Ichim, L.: Using fractal dimension in tumor growth evaluation. In: Proceedings of the 5th WSEAS International Conference on Non-linear Analysis, Non-linear systems and chaos, pp. 63-68. World Scientific and Engineering Academy and Society (WSEAS) (2006)

8. Gneiting, T., Ševčíková, H., Percival, D.B.: Estimators of fractal dimension: assessing the roughness of time series and spatial data. Stat. Sci. 27, 247-277 (2012)

9. Keough, K.M.W., Hyam, P., Pink, D.A., Quinn, B.: Cell surfaces and fractal dimensions. J. Microsc. 163(1), 95-99 (1991)

10. Mandelbrot, B.B.: The Fractal Geometry of Nature/Revised and Enlarged Edition, p. 495. WH Freeman and Co., New York (1983)

11. Goh, C., Hamadicharef, B., Henderson, G., Ifeachor, E.: Comparison of fractal dimension algorithms for the computation of EEG biomarkers for dementia. In: 2nd International Conference on Computational Intelligence in Medicine and Healthcare (CIMED2005) (2005) 
12. Molina-Abril, H., Real, P., Nakamura, A., Klette, R.: Connectivity calculus of fractal polyhedrons. Pattern Recognit. 48(4), 1150$1160(2015)$

13. Molina-Abril, H., Real, P.: Homological spanning forest framework for 2D image analysis. Ann. Math. Artif. Intell. 64(4), 385-409 (2012)

14. Napolitano, A., Ungania, S., Cannata, V.: Fractal dimension estimation methods for biomedical images. In: MATLAB-A Fundamental Tool for Scientific Computing and Engineering Applications-Volume 3. InTech (2012)

15. Real, P., Molina-Abril, H., Gonzalez-Lorenzo, A., Bac, A., Mari, J.L.: Searching combinatorial optimality using graph-based homology information. Appl. Algebra Eng. Commun. Comput. 26(1-2), 103-120 (2015)

16. Rosenfeld, A.: Adjacency in Digital Pictures. Inf. Control 26, 24-33 (1974)

17. Serra, J.: Image Analysis and Mathematical Morphology. Academic Press, Cambridge (1982)

18. Smith Jr., T.G., Lange, G.D., Marks, W.B.: Fractal methods and results in cellular morphology dimensions, lacunarity and multifractals. J. Neurosci. Methods 69(2), 123-136 (1996)

19. Timbo, C., da Rosa, L.A.R., Goncalves, M., Duarte, S.B.: Computational cancer cells identification by fractal dimension analysis. Comput. Phys. Commun. 180(6), 850-853 (2009)

20. Uppal, S.O., Voronine, D.V., Wendt, E., Heckman, C.A.: Morphological fractal analysis of shape in cancer cells treated with combinations of microtubule-polymerizing and-depolymerizing agents. Microsc. Microanal. 16(4), $472-477$ (2010)

21. Venkatesh, B., Chen, D.L., Bhole, S.D.: Three-dimensional fractal analysis of fracture surfaces in a titanium alloy for biomedical applications. Scripta Materialia 59(4), 391-394 (2008)

22. Voss, R.F.: Random fractals: characterization and measurement. In: Scaling phenomena in disordered systems, pp. 1-11. Springer, Boston (1991) 